\title{
Which Cayley graphs are integral?
}

\author{
A. Abdollahi \\ Department of Mathematics \\ University of Isfahan \\ Isfahan 81746-73441 \\ Iran \\ a.abdollahi@math.ui.ac.ir
}

\author{
E. Vatandoost \\ Department of Mathematics \\ University of Isfahan \\ Isfahan 81746-73441 \\ Iran \\ e.vatandoost@math.ui.ac.ir
}

Submitted: Mar 7, 2009; Accepted: Sep 14, 2009; Published: Sep 25, 2009

Mathematics Subject Classifications: 05C25; 05C50

\begin{abstract}
Let $G$ be a non-trivial group, $S \subseteq G \backslash\{1\}$ and $S=S^{-1}:=\left\{s^{-1} \mid s \in S\right\}$. The Cayley graph of $G$ denoted by $\Gamma(S: G)$ is a graph with vertex set $G$ and two vertices $a$ and $b$ are adjacent if $a b^{-1} \in S$. A graph is called integral, if its adjacency eigenvalues are integers. In this paper we determine all connected cubic integral Cayley graphs. We also introduce some infinite families of connected integral Cayley graphs.
\end{abstract}

\section{Introduction and Results}

We say that a graph is integral if all the eigenvalues of its adjacency matrix are integers. The notion of integral graphs was first introduced by Harary and Schwenk in 1974 [12].

In 1976 Bussemaker and Cvetković [7], proved that there are exactly 13 connected cubic integral graphs. The same result was independently proved by Schwenk [16] who unlike the effort in [7] avoids the use of computer search to examine all the possibilities. However the work of Schwenk [16] was inspired and stimulated by Cvetković attempt [9] to find the connected cubic integral graphs where he had displayed twelve such graphs, and had restricted the remaining possibilities to ninety-five potential spectra, Schwenk has produced a complete and self-contained solution.

It is known that the size of a connected $k$-regular graph with diameter $d$ is bounded above by $\frac{k(k-1)^{d}-2}{k-2}$ (see, for example [10]). In [9], it is noted that if we know the graph is integral then $d \leqslant 2 k$ because there are at most $2 k+1$ distinct eigenvalues. Consequently, the upper bound of the size of a connected $k$-regular integral graph is

$$
n \leqslant \frac{k(k-1)^{2 k}-2}{k-2}
$$


Using Brendan McKay's program geng for generating graphs, nowadays it is easy to see that there are exactly 263 connected integral graphs on up to 11 vertices (see $[3,4])$. In 2009 Alon et al. [1] show that the total number of adjacency matrices of integral graphs with $n$ vertices is less than or equal to $2^{\frac{n(n-1)}{2}}-\frac{n}{400}$ for a sufficiently large $n$. For the background and some known results about integral graphs, we refer the reader to the survey [5].

The problem of characterizing integral graphs seems to be very difficult and so it is wise to restrict ourselves to certain families of graphs. Here we are interested to study Cayley graphs. Let $G$ be a non-trivial group with the identity element $1, S \subseteq G \backslash\{1\}$ and $S=S^{-1}:=\left\{s^{-1} \mid s \in S\right\}$. The Cayley graph of $G$ denoted by $\Gamma(S: G)$ is the graph with vertex set $G$ and two vertices $a$ and $b$ are adjacent if $a b^{-1} \in S$. If $S$ generates $G$ then $\Gamma(S: G)$ is connected. A Cayley graph is simple and vertex transitive.

We denote the symmetric group and the alternating group on $n$ letters by $S_{n}$ and $A_{n}$, respectively. Also $C_{m}$ and $D_{2 n}$ are used for the cyclic group of order $m$ and dihedral group of order $2 n(n>2)$.

The main question that we are concerned here is the following:

Which Cayley graphs are integral?

It is clear that if $S=G \backslash\{1\}$, then $\Gamma(S: G)$ is the complete graph with $|G|$ vertices and so it is integral. Klotz and Sander [14] showed that all nonzero eigenvalues of $\Gamma\left(U_{n}: \mathbb{Z}_{n}\right)$ are integers dividing the value $\varphi(n)$ of the Euler totient function, where $\mathbb{Z}_{n}$ is the cyclic group of order $n$ and $U_{n}$ is the subset of all elements of $\mathbb{Z}_{n}$ of order $n$. W. So [17] characterize integral graphs among circulant graphs. By using a result of Babai [2] which presents the spectrum of a Cayley graph in terms of irreducible characters of the underlying group, we give some infinite families of integral Cayley graphs.

The study of Cayley graphs of the symmetric group generated by transpositions is interesting (See [11]). In this paper we show $\Gamma\left(S: S_{n}\right)$ is integral, where $S=\{(12),(13), \ldots,(1 n)\}$ and $n \in\{3,4,5,6\}$. We also characterize all connected cubic integral Cayley graphs and introduce some infinite family of connected integral Cayley graphs.

The main results are the following.

Theorem 1.1 There are exactly seven connected cubic integral Cayley graphs. In particular, for a finite group $G$ and a subset $S=S^{-1} \not \supset 1$ with three elements, $\Gamma(S: G)$ is integral if and only if $G$ is isomorphic to one the following groups: $C_{2}^{2}, C_{4}, C_{6}, S_{3}, C_{2}^{3}$, $C_{2} \times C_{4}, D_{8}, C_{2} \times C_{6}, D_{12}, A_{4}, S_{4}, D_{8} \times C_{3}, D_{6} \times C_{4}$ or $A_{4} \times C_{2}$.

Theorem 1.2 Let $D_{2 n}=\left\langle a, b \mid a^{n}=b^{2}=1,(a b)^{2}=1\right\rangle, n=2 m+1, d \mid n(1<d<n)$ and $S=\left\{a^{k} \mid k \in B(1, n)\right\} \cup\left\{a^{d k} \mid k \in B\left(1, \frac{n}{d}\right)\right\} \cup\left\{b a^{k} \mid k \in B(1, n)\right\} \cup\left\{b a^{d k} \mid k \in\right.$ $\left.B\left(1, \frac{n}{d}\right)\right\}$. Then $\Gamma\left(S: D_{2 n}\right)$ is integral.

Theorem 1.3 Let $T_{4 n}=\left\langle a, b \mid a^{2 n}=1, b^{2}=a^{n}, b^{-1} a b=a^{-1}\right\rangle, n=2 m+1(n \neq 1)$ and $S=\left\{a^{k} \mid 1 \leqslant k \leqslant 2 n-1, k \neq n\right\} \cup\left\{a b, a^{n+1} b\right\}$. Then $\Gamma\left(S: T_{4 n}\right)$ is integral.

Theorem 1.4 Let $U_{6 n}=\left\langle a, b \mid a^{2 n}=b^{3}=1, a^{-1} b a=b^{-1}\right\rangle, n=2 m+1(n \neq 1)$ and $S=\left\{a^{2 k} b \mid 1 \leqslant k \leqslant n-1\right\} \cup\left\{a^{2 k} b^{2} \mid 1 \leqslant k \leqslant n-1\right\} \cup\left\{a^{2 k+1} b \mid 0 \leqslant k \leqslant n-1\right\}$. Then $\Gamma\left(S: U_{6 n}\right)$ is integral. 


\section{Preliminaries}

First we give some facts that are needed in the next section. Let $n$ be a positive integer. Then $B(1, n)$ denotes the set $\{j \mid 1 \leqslant j<n,(j, n)=1\}$. Let $\omega=e^{\frac{2 \pi i}{n}}$ and

$$
C(r, n)=\sum_{j \in B(1, n)} \omega^{j r}, \quad 0 \leqslant r \leqslant n-1
$$

The function $C(r, n)$ is a Ramanujan sum. For integers $r$ and $n,(n>0)$, Ramanujan sums have only integral values ( See [15] and [18]).

Lemma 2.1 Let $\omega=e^{\frac{\pi i}{n}}$, where $i^{2}=-1$. Then

i) $\sum_{j=1}^{2 n-1} \omega^{j}=-1$.

ii) If $l$ is even, then $\sum_{j=1}^{n-1} \omega^{l j}=-1$.

iii) If $l$ is odd, then $\sum_{j=1}^{n-1} \omega^{l j}+\omega^{-l j}=0$.

Proof. The proof is straightforward.

Lemma 2.2 Let $G=C_{n}=\langle a\rangle, d \mid n(1<d<n)$ and $A_{d}=\left\{a^{d k} \mid k \in B\left(1, \frac{n}{d}\right)\right\}$. Then $A_{d}^{-1}=A_{d}$.

Proof. Let $n=d k^{\prime}$ and $a^{d k}$ be an arbitrary element of $A_{d}$. Since $\left(k-k^{\prime}, k^{\prime}\right)=1$ and $\left(a^{d k}\right)^{-1}=a^{n-d k}=a^{d k^{\prime}-d k}=a^{\left(k^{\prime}-k\right) d},\left(a^{d k}\right)^{-1} \in A_{d}$. So $A_{d}^{-1} \subseteq A_{d}$. It is easy to see that $\left|A_{d}^{-1}\right|=\left|A_{d}\right|$. Hence $A_{d}^{-1}=A_{d}$.

Lemma 2.3 [2] Let $G$ be a finite group of order $n$ whose irreducible characters (over $\mathbb{C})$ are $\rho_{1}, \ldots, \rho_{h}$ with respective degrees $n_{1}, \ldots, n_{h}$. Then the spectrum of the Cayley graph $\Gamma(S: G)$ can be arranged as $\Lambda=\left\{\lambda_{i j k} \mid i=1, \ldots, h ; j, k=1, \ldots, n_{i}\right\}$ such that $\lambda_{i j 1}=\ldots=\lambda_{i j n_{i}}$ and

$$
\lambda_{i 1}^{t}+\ldots+\lambda_{i n_{i}}^{t}=\sum_{s_{1}, \ldots, s_{t} \in S} \rho_{i}\left(\Pi_{l=1}^{t} s_{l}\right),
$$

for any natural number $t$.

Lemma 2.4 [13] Let $C_{n}=\langle a\rangle$. Then irreducible characters of $C_{n}$ are $\rho_{j}: a^{k} \mapsto \omega^{j k}$, where $j, k=0,1, \ldots, n-1$.

Lemma 2.5 [13] Let $G=C_{n_{1}} \times \cdots \times C_{n_{r}}$ and $C_{n_{i}}=\left\langle a_{i}\right\rangle$, so that for any $i, j \in\{1, \ldots, r\}$, $\left(n_{i}, n_{j}\right) \neq 1$. If $\omega_{t}=e^{\frac{2 \pi i}{n_{t}}}$, then $n_{1} \cdots n_{r}$ irreducible characters of $G$ are

$$
\rho_{l_{1} \ldots l_{r}}\left(a_{1}^{k_{1}}, \ldots, a_{r}^{k_{r}}\right)=\omega_{1}^{l_{1} k_{1}} \omega_{2}^{l_{2} k_{2}} \cdots \omega_{r}^{l_{r} k_{r}}
$$

where $l_{i}=0,1, \ldots, n_{i}-1$ and $i=1,2, \ldots, r$. 
Lemma 2.6 Let $G$ be a group and $G=\langle S\rangle$, where $S=S^{-1}$ and $1 \notin S$. If $a \in S$ and $o(a)=m>2$, then $\Gamma(S: G)$ has the cycle with $m$ vertices as a subgraph.

Proof. Observe that $1-a-a^{2}-\cdots-a^{m-1}-a^{m}=1$ is a cycle with $m$ vertices.

Lemma 2.7 Let $G=\langle S\rangle$ be a group, $|G|=n,|S|=2, S=S^{-1} \not \supset 1$. Then $\Gamma(S: G)$ is integral if and only if $n \in\{3,4,6\}$.

Proof. It is clear that $\Gamma(S: G)$ is a connected 2-regular graph. Thus $\Gamma(S: G)$ is the cycle with $n$ vertices. By checking the eigenvalues of the cycles, one can easily see that the only integral cycles are ones with 3,4 or 6 vertices. This completes the proof.

Lemma 2.8 Let $G$ be the cyclic group $\langle a\rangle,|G|=n>3$ and let $S$ be a generating set of $G$ such that $|S|=3, S=S^{-1}$ and $1 \notin S$. Then $a^{n / 2} \in S$. Also if $a^{r} \in S$ and $o\left(a^{r}\right)=m>2$, then $(n, r)=1$ or $(n / 2, r)=1$.

Proof. Let $(n, r) \neq 1$ and $(n / 2, r) \neq 1$. Then $\left\langle a^{r}\right\rangle \neq G$. Suppose $(n / 2, r)=d$, where $d \neq 1$, then $\left\langle a^{r}, a^{n / 2}\right\rangle=\left\langle a^{d}\right\rangle$. Since $d \mid n, G \neq\left\langle a^{d}\right\rangle$. Hence $\left\langle a^{r}, a^{n / 2}\right\rangle \neq G$. This contradicts the fact that $S$ generates $G$.

Lemma 2.9 Let $G$ be the cyclic group $\langle a\rangle,|G|=n>3$ and let $S$ be a generating set of $G$ such that $|S|=3, S=S^{-1}$ and $1 \notin S$. Then $\Gamma(S: G)$ is integral if and only if $n \in\{4,6\}$.

Proof. Let $\Gamma(S: G)$ be integral. Then $S=\left\{a^{n / 2}, a^{r}, a^{-r}\right\}$, where $(n, r)=1$ or $(n / 2, r)=$ 1. If $\lambda$ is the eigenvalue of $\Gamma(S: G)$ corresponding to irreducible character of $\rho_{1}$. Then by Lemmas 2.3 and $2.4, \lambda=\rho_{1}\left(a^{r}\right)+\rho_{1}\left(a^{-r}\right)+\rho_{1}\left(a^{n / 2}\right)=2 \cos (2 \pi r / n)-1$. Since $\lambda$ is integer, $\cos (2 \pi r / n) \in\{ \pm 1 / 2, \pm 1,0\}$. We consider the following cases:

Case1: Let $(n, r)=1$. Then if $\cos (2 \pi r / n) \in\{-1 / 2,-1,1\}$, then $n \in\{1,2,3\}$, which is false. If $\cos (2 \pi r / n)=0$, then $n=4$ and $r=1$ or 3 . So $S=\left\{a, a^{2}, a^{3}\right\}$. If $\cos (2 \pi r / n)=$ $1 / 2$, then $n=6$ and $r=1$ or 5 . So $S=\left\{a, a^{3}, a^{5}\right\}$.

Case2: Let $(n, r) \neq 1$ and $(n / 2, r)=1$. Without loss of generality we can assume $r<n / 2$. Similarly if $\cos (2 \pi r / n) \in\{-1,0,1 / 2,1\}$, then $r=1$, which is false. If $\cos (2 \pi r / n)=-1 / 2$, then $n=6$ and $r=2$ or 4 . So $S=\left\{a^{2}, a^{3}, a^{4}\right\}$.

Conversely, if $n=4$, then $\Gamma(S: G)$ is complete graph $K_{4}$ and so is integral.

If $n=6, S_{1}=\left\{a, a^{3}, a^{5}\right\}$ and $S_{2}=\left\{a^{2}, a^{3}, a^{4}\right\}$, then by Lemmas 2.3 and $2.4, \Gamma\left(S_{1}: G\right)$ and $\Gamma\left(S_{2}: G\right)$ are integral with spectra of $\left[-3,0^{4}, 3\right]$ and $\left[-2^{2}, 0^{2}, 1,3\right]$ respectively.

Lemma 2.10 Let $G_{1}$ and $G_{2}$ be two groups and $G=G_{1} \times G_{2}$ such that $\Gamma(S: G)$ is integral, where $S=S^{-1} \not \ngtr 1$ with three elements. Let $S_{1}=\left\{s_{1} \mid\left(s_{1}, g_{2}\right) \in S, g_{2} \in\right.$ $\left.G_{2}\right\} \backslash\{1\}$. Then $\Gamma\left(S_{1}: G_{1}\right)$ is integral.

Proof. Let $\chi_{0}$ and $\rho_{0}$ be the trivial irreducible characters of $G_{1}$ and $G_{2}$, respectively. Let $\lambda_{i 0}$ and $\lambda_{i}$ be the eigenvalues of $\Gamma(S: G)$ and $\Gamma\left(S_{1}: G_{1}\right)$ corresponding to irreducible 
characters of $\chi_{i} \times \rho_{0}$ and $\chi_{i}$, respectively. Since $S$ generates $G$ and $S=S^{-1} \not \ngtr 1$ with three elements, $\left|S_{1}\right|=2$ or 3 . If $\left|S_{1}\right|=2$, then by Lemma 2.3,

$$
\lambda_{i 0}=\sum_{\left(g_{1}, g_{2}\right) \in S}\left(\chi_{i} \times \rho_{0}\right)\left(g_{1}, g_{2}\right)=\sum_{s_{1} \in S_{1}} \chi_{i}\left(s_{1}\right)+1
$$

and so $\lambda_{i 0}=\lambda_{i}+1$. If $\left|S_{1}\right|=3$, then by Lemma 2.3 ,

$$
\lambda_{i 0}=\sum_{\left(g_{1}, g_{2}\right) \in S}\left(\chi_{i} \times \rho_{0}\right)\left(g_{1}, g_{2}\right)=\sum_{s_{1} \in S_{1}} \chi_{i}\left(s_{1}\right)=\lambda_{i}
$$

and so $\operatorname{Spec}\left(\Gamma\left(S_{1}: G_{1}\right)\right) \subseteq \operatorname{Spec}(\Gamma(S: G))$. However $\Gamma\left(S_{1}: G_{1}\right)$ is integral. Furthermore if $\left|S_{1}\right|=2$, then $-1 \leqslant \lambda_{i 0}$.

Lemma 2.11 Let $G$ be a finite abelian group such that is not cyclic and let $G=\langle S\rangle$, where $|S|=3, S=S^{-1}$ and $1 \notin S$. Then $\Gamma(S: G)$ is integral if and only if $|G| \in\{4,8,12\}$.

Proof. Let $\Gamma(S: G)$ be integral. If all of elements of $S$ are of order two, then $G=C_{2}^{2}$ or $G=C_{2}^{3}$. So $|G|=4$ or 8 . Otherwise $G=C_{m} \times C_{2}$ where $m$ is even. By Lemmas $2.7,2.9$ and 2.10 , we conclude that $m \in\{3,4,6\}$. Since $m$ is even, $m \in\{4,6\}$. Hence $|G| \in\{4,8,12\}$.

Conversely, if $|G|=4$, then $\Gamma(S: G)=K_{4}$ and so is integral.

Let $|G|=8$. Then $G=C_{2}^{3}$ or $C_{4} \times C_{2}$. If $G=C_{2}^{3}$ and $S=\{(b, 1,1),(1, b, 1),(1,1, b)\}$, then by Lemma 2.3, $\Gamma\left(S: C_{2}^{3}\right)$ is integral with spectrum of $\left[-3,-1^{3}, 1^{3}, 3\right]$. If $G=C_{4} \times C_{2}$ and $S=\left\{(a, 1),\left(a^{3}, 1\right),(1, b)\right\}$, then by Lemma $2.3, \Gamma\left(S: C_{4} \times C_{2}\right)$ is integral with spectrum of $\left[-3,-1^{3}, 1^{3}, 3\right]$.

Let $|G|=12$. Then $G=C_{6} \times C_{2}$. If $S=\left\{(a, 1),\left(a^{5}, 1\right),(1, b)\right\}$, then by Lemma 2.3, $\Gamma\left(S: C_{6} \times C_{2}\right)$ is integral with spectrum of $\left[-3,-2^{2},-1,0^{4}, 1,2^{2}, 3\right]$.

Lemma 2.12 Let $D_{2 n}=\left\langle a, b \mid a^{n}=b^{2}=1,(a b)^{2}=1\right\rangle, n=2 m+1$, and $\Gamma\left(S: D_{2 n}\right)$ be integral, where $D_{2 n}=\langle S\rangle,|S|=3, S=S^{-1}$ and $1 \notin S$. Then

i) -3 is the simple eigenvalue of $\Gamma\left(S: D_{2 n}\right)$ if and only if all of elements of $S$ are of order two.

ii) If $\left[-3,-2^{l_{1}},-1^{l_{2}}, 0^{l_{3}}, 1^{l_{4}}, 2^{l_{5}}, 3\right]$ is the spectrum of $\Gamma\left(S: D_{2 n}\right)$, then $l_{1}=l_{4}, l_{2}=l_{5}$ and $4 \mid l_{3}$. Furthermore $l_{1}, l_{2}$ are even.

iii) If $n \neq 3$, then $\Gamma\left(S: D_{2 n}\right)$ is bipartite.

Proof. i) Let -3 be the simple eigenvalue of $\Gamma\left(S: D_{2 n}\right)$. By Lemma 2.3 and using characters table $D_{2 n},-3$ is the eigenvalue of $\Gamma\left(S: D_{2 n}\right)$ corresponding to irreducible character $\chi_{m+1}$. So all of elements of $S$ are in conjugacy class of $b$.

Conversely, if all of elements of $S$ are of order two, then $S \subseteq \bar{b}$ (the bar indicates conjugacy class). By Lemma 2.3 and using characters table of $D_{2 n}$, the eigenvalue of $\Gamma\left(S: D_{2 n}\right)$ corresponding to irreducible character $\chi_{m+1}$ is -3 . 
ii) Since -3 is the simple eigenvalue of $\Gamma\left(S: D_{2 n}\right), S \subseteq \bar{b}$. By Lemma 2.3 and using characters table of $D_{2 n}$, the eigenvalues of $\Gamma\left(S: D_{2 n}\right)$ corresponding to irreducible characters $\chi_{j}(1 \leqslant j \leqslant m)$, are negative. Thus $l_{1}=l_{4}$ and $l_{2}=l_{5}$. Furthermore since the multiplicity of the eigenvalues of corresponding to irreducible characters of degree two is $2, l_{1}$ and $l_{2}$ are even and $4 \mid l_{3}$.

iii) Let $a^{r} \in S$, where $1 \leqslant r \leqslant m$. It is clear that $(n, r)=1$. Since $n \neq 3$ and $(n, r)=1,2 \cos (2 \pi r / n)$ is not integer. Let $\lambda_{11}$ and $\lambda_{12}$ be eigenvalues of $\Gamma\left(S: D_{2 n}\right)$ corresponding to irreducible character $\chi_{1}$. By Lemma 2.3 and using characters table of $D_{2 n}, \lambda_{11}+\lambda_{12}=2 \cos (2 \pi r / n)$. This contradicts the fact that $\Gamma\left(S: D_{2 n}\right)$ is integral. Thus $S \subseteq \bar{b}$ and so -3 is an eigenvalue of $\Gamma\left(S: D_{2 n}\right)$. Therefore, $\Gamma\left(S: D_{2 n}\right)$ is bipartite.

Lemma 2.13 Let $S=\{(12),(13), \ldots,(1 n)\}$ and $n \in\{3,4,5,6\}$. Then $\Gamma\left(S: S_{n}\right)$ is integral.

Proof. It is clear that $\Gamma\left(S: S_{3}\right)$ is a cycle with six vertices and so is integral with spectrum of $\left[-2,-1^{2}, 1^{2}, 2\right]$. By using the following program written in GAP [19] and thanks to the GRAPE package of L.H. Soicher, one can easily see that $\Gamma\left(S: S_{4}\right), \Gamma\left(S: S_{5}\right)$ and $\Gamma\left(S: S_{6}\right)$ are integral graphs with spectra as follows:

$$
\begin{gathered}
{\left[-3,-2^{6},-1^{3}, 0^{4}, 1^{3}, 2^{6}, 3\right],} \\
{\left[-4,-3^{12},-2^{28},-1^{4}, 0^{30}, 1^{4}, 2^{28}, 3^{12}, 4\right],} \\
{\left[-5,-4^{20},-3^{105},-2^{120},-1^{30}, 0^{168}, 1^{30}, 2^{120}, 3^{105}, 4^{20}, 5\right],}
\end{gathered}
$$

respectively.

LoadPackage ("grape");

\#\#\# The following function admat constructs the adjacency matrix

\#\#\# of a given graph $G$ with $n$ vertices

admat: $=$ function $(\mathrm{G}, \mathrm{n})$

local $B, A, i, j$;

$A:=[]$;

for $i$ in $[1 \ldots n]$ do

$B:=[]$;

for $j$ in $[1 \ldots n]$ do

if ( $j$ in $\operatorname{Adjacency}(G, i))=$ true then $\operatorname{Add}(B, 1)$; else

$\operatorname{Add}(B, 0)$; fi;

od;

$\operatorname{Add}(A, B)$;

od;

return A;

end;

\#\#\# The following function listcompress converts a multiset to a set

\#\#\# of ordered pairs whose first components are exactly the

\#\#\# elements of the corresponding set to the multiset 


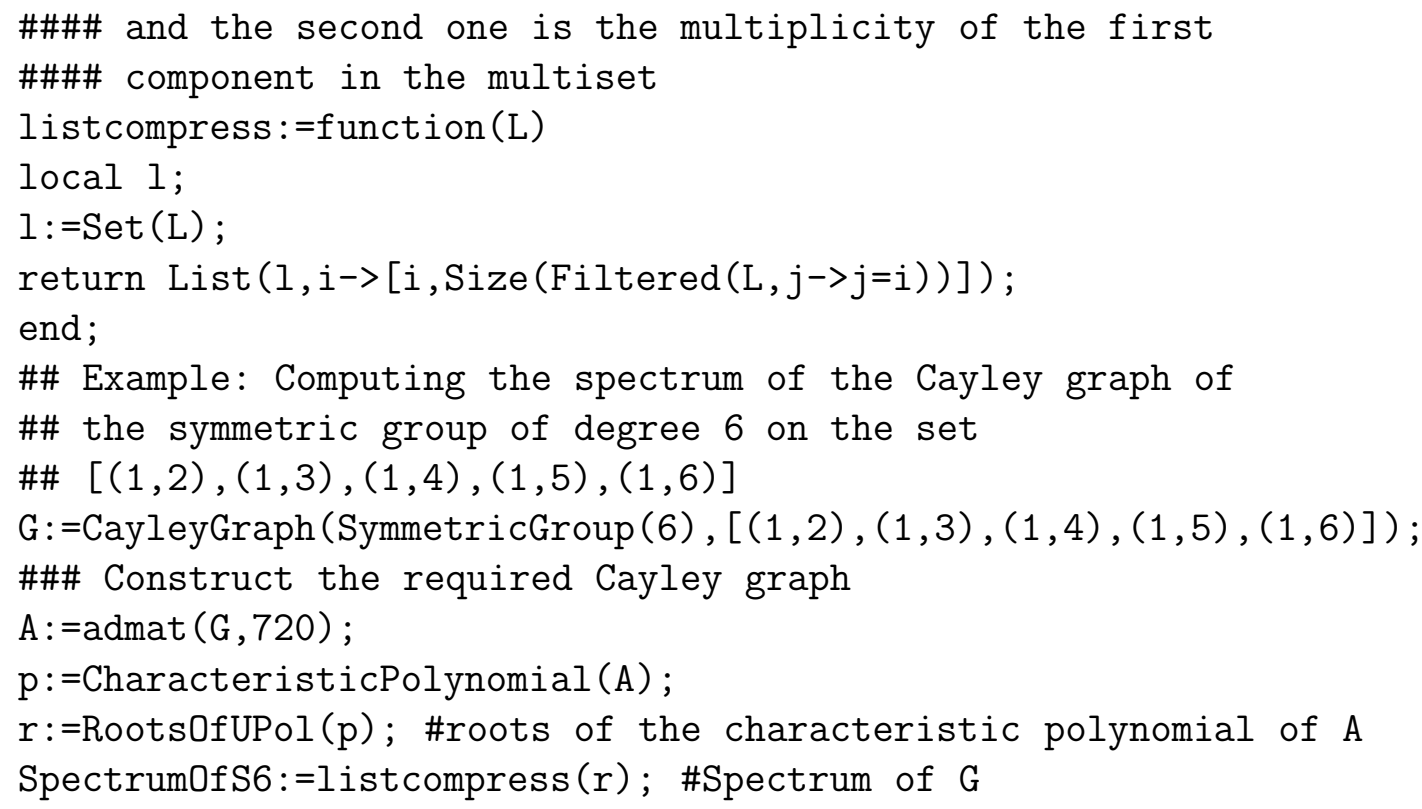

We end this section by the following conjecture.

Conjecture 2.14 Let $n \geqslant 4$ be an arbitrary integer and $S=\{(12),(13), \ldots,(1 n)\}$ be the subset of the symmetric group $S_{n}$ of degree $n$. Then $\Gamma\left(S: S_{n}\right)$ is integral. Moreover, $\{0, \pm 1, \ldots, \pm(n-1)\}$ is the set of all distinct eigenvalues of $\Gamma\left(S: S_{n}\right)$.

\section{Proof of Our main results}

In this section we prove our main results.

Proof of Theorem 1.1. Let $\Gamma(S: G)$ be integral. Since $\Gamma(S: G)$ is a cubic integral graph, $\Gamma(S: G)$ is of type $G_{i}$, for $1 \leqslant i \leqslant 13$ (see [16]). Since the number of vertices of $G_{i}$, for $1 \leqslant i \leqslant 13$, are $4,6,8,10,12,20,24$ or $30,|G| \in\{4,6,8,10,12,20,24,30\}$. Hence we have the following cases:

Case1: Let $|G|=4$. Then $\Gamma(S: G)=K_{4}=G_{1}$.

Case2: Let $|G|=6$. Then $G=C_{6}$ or $D_{6}$.

If $C_{6}=\langle a\rangle, S_{1}=\left\{a, a^{3}, a^{5}\right\}$ and $S_{2}=\left\{a^{2}, a^{3}, a^{4}\right\}$, then by using the program written in Lemma 2.13, $\Gamma\left(S_{1}: C_{6}\right)$ and $\Gamma\left(S_{2}: C_{6}\right)$ are integral with spectra of $\left[-3,0^{4}, 3\right]$ and $\left[-2^{2}, 0^{2}, 1,3\right]$ respectively. So $\Gamma\left(S_{1}: C_{6}\right)=G_{2}$ and $\Gamma\left(S_{2}: C_{6}\right)=G_{5}$.

If $G=D_{6}=\left\langle a, b \mid a^{3}=b^{2}=(a b)^{2}=1\right\rangle, S_{1}=\left\{b, a b, a^{2} b\right\}$ and $S_{2}=\left\{a, a^{2}, b\right\}$, then by using the program written in Lemma $2.13, \Gamma\left(S_{1}: D_{6}\right)$ and $\Gamma\left(S_{2}: D_{6}\right)$ are integral with spectra of $\left[-3,0^{4}, 3\right]$ and $\left[-2^{2}, 0^{2}, 1,3\right]$ respectively. So $\Gamma\left(S_{1}: D_{6}\right)=G_{2}$ and $\Gamma\left(S_{2}: D_{6}\right)=G_{5}$.

Case3: Let $|G|=8$. Then $G=C_{8}, C_{2}^{3}, C_{4} \times C_{2}, D_{8}$ or $Q_{8}=\langle a, b| a^{4}=1, a^{2}=$ $\left.b^{2}, b^{-1} a b=a^{-1}\right\rangle$. We show that the graph $G_{4}$ is only and only cayley graph of $C_{2}^{3}, C_{4} \times C_{2}$ and $D_{8}$. 
Let $G=C_{2}^{3}$ or $C_{4} \times C_{2}$, by the proof of Lemma $2.11, \Gamma(S: G)=G_{4}$.

Let $G=D_{8}=\left\langle a, b \mid a^{4}=b^{2}=(a b)^{2}=1\right\rangle$ and $S=\left\{a, a^{-1}, b\right\}$ or $\left\{b, a^{2} b, a b\right\}$. Then by using the program written in Lemma $2.13, \Gamma\left(S: D_{8}\right)$ is integral with spectrum of $\left[-3,-1^{3}, 1^{3}, 3\right]$ and so $\Gamma\left(S: D_{8}\right)=G_{4}$.

Let $G=C_{8}$. By Lemma 2.9, $\Gamma\left(S: C_{8}\right)$ is not isomorphic to $G_{4}$.

Let $G=Q_{8}$. Since $a^{2}$ is the unique element of degree two, $a^{2} \in S$. Since $S$ is generator and $S=S^{-1}, S=\left\{a^{2}, b, a^{2} b\right\}$ or $\left\{a^{2}, a b, a^{3} b\right\}$. If $S=\left\{a^{2}, b, a^{2} b\right\}$, then by Lemma 2.3 and using characters table of $Q_{8}$, the eigenvalue of $\Gamma\left(S: Q_{8}\right)$ corresponding to the irreducible character $\chi_{3}$ is 3 . If $S=\left\{a^{2}, a b, a^{3} b\right\}$, then the eigenvalue of $\Gamma\left(S: Q_{8}\right)$ corresponding to the irreducible character $\chi_{4}$ is 3 . However the multiplicity 3 as an eigenvalue of $\Gamma\left(S: Q_{8}\right)$ is greater than one. So $\Gamma\left(S: Q_{8}\right)$ is not isomorphic to $G_{4}$.

Case4: Let $|G|=10$. Then by Lemmas 2.9 and 2.11, $G$ is a non-abelian group and so $G=D_{10}$. Since $\Gamma\left(S: D_{10}\right)$ is integral, $\Gamma\left(S: D_{10}\right)=G_{3}, G_{7}$ or $G_{11}$. If $\Gamma\left(S: D_{10}\right)=G_{3}$ or $G_{7}$, then $\Gamma\left(S: D_{10}\right)$ is not bipartite graph, which by Lemma 2.12 (iii), is a contradiction. If $\Gamma\left(S: D_{10}\right)=G_{11}$, then by Lemma $2.12(i i)$, it is a contradiction. Therefore, the graphs of $G_{3}, G_{7}$ and $G_{11}$ are not Cayley graphs.

Case5: Let $|G|=12$. By Lemmas 2.9 and 2.11, $G=C_{6} \times C_{2}, T_{12}, A_{4}$ or $D_{12}$. First we show $G_{12}$ is only and only cayley graph of $C_{6} \times C_{2}$ and $D_{12}$.

Let $G=C_{6} \times C_{2}$ and $S=\left\{(a, c),\left(a^{-1}, c\right),\left(a^{3}, c\right)\right\}$ where $C_{6}=\langle a\rangle$ and $C_{2}=\langle c\rangle$. Then by using the program written in Lemma 2.13, $\Gamma\left(S: C_{6} \times C_{2}\right)$ is integral with spectrum of $\left[-3,-2^{2},-1,0^{4}, 1,2^{2}, 3\right]$. So $\Gamma\left(S: C_{6} \times C_{2}\right)=G_{12}$.

Let $G=D_{12}=\left\langle a, b \mid a^{6}=b^{2}=(a b)^{2}=1\right\rangle$ and $S=\left\{a, a^{5}, b\right\}$. Then by using the program written in Lemma $2.13, \Gamma\left(S: D_{12}\right)$ is integral with spectrum of $\left[-3,-2^{2},-1,0^{4}, 1,2^{2}, 3\right]$. So $\Gamma\left(S: D_{12}\right)=G_{12}$.

Let $\Gamma\left(S: T_{12}\right)=G_{12}$. It is easy to see that $a^{3}$ is the unique element of order two, so $a^{3} \in S$. Since $S$ generates $G, a^{r} \notin S$. By Lemma 2.3 and using characters table of $T_{4 n}$, we conclude that the eigenvalues of $\Gamma\left(S: T_{12}\right)$ corresponding to linear irreducible characters of $T_{12}$ are distinct from -3 . Therefore, $G_{12}$ have not -3 as an eigenvalue, which is not true. So $G_{12}$ is not Cayley graph of $T_{12}$.

Let $\Gamma\left(S: A_{4}\right)=G_{12}$. By Lemma 2.3 and using characters table of $A_{4}, \Gamma\left(S: A_{4}\right)$ has an eigenvalue with multiplicity greater than 6 or three eigenvalues with multiplicities greater than 3. Which is impossible.

Therefor the graph $G_{12}$ is only and only Cayley graph of $C_{6} \times C_{2}$ and $D_{12}$.

We continue by showing that $G_{8}$ is only and only Cayley graph $A_{4}$.

Let $G=A_{4}$ and $S=\left\{\left(\begin{array}{ll}1 & 2\end{array}\right)\left(\begin{array}{ll}3 & 4\end{array}\right),\left(\begin{array}{lll}1 & 2 & 3\end{array}\right),\left(\begin{array}{lll}1 & 3 & 2\end{array}\right)\right\}$. By using the program written in Lemma 2.13, $\Gamma\left(S: A_{4}\right)$ is integral with spectrum of $\left[-2^{3},-1^{3}, 0^{2}, 2^{3}, 3\right]$, and so $\Gamma\left(S: A_{4}\right)=G_{8}$.

Let $\Gamma\left(S: T_{12}\right)=G_{8}$. Since $G_{8}$ does not have $C_{4}$ as a subgraph, by Lemma 2.6, $S=\left\{a^{3}, a^{r}, a^{-r}\right\}$ for $r=1,2$. This contradicts the fact that $S$ generates $G$.

Let $\Gamma\left(S: C_{6} \times C_{2}\right)=G_{8}$ and $S_{1}=\left\{s_{1} \mid\left(s_{1}, c\right) \in S, c \in C_{2}\right\} \backslash\{1\}$. Then by Lemma 2.10 and case $2,\left|S_{1}\right|=2$ and so $-1 \leqslant \lambda_{i 0}$, where $\lambda_{i 0}$ is the eigenvalue of $\Gamma\left(S: C_{6} \times C_{2}\right)$ corresponding to a linear irreducible character of $C_{6} \times C_{2}$. This contradicts the fact that -2 is an eigenvalue of $G_{8}$. 
Let $\Gamma\left(S: D_{6} \times C_{2}\right)=G_{8}$ and $S_{1}=\left\{s_{1} \mid\left(s_{1}, c\right) \in S, c \in C_{2}\right\} \backslash\{1\}$. Then by Lemma 2.10 and case $2,\left|S_{1}\right|=2$ and so $-1 \leqslant \lambda_{i 0}$, where $\lambda_{i 0}$ is the eigenvalue of $\Gamma\left(S: D_{6} \times C_{2}\right)$ corresponding to a linear irreducible character of $D_{6} \times C_{2}$. This contradicts the fact that -2 is an eigenvalue of $G_{8}$.

Therefor the graph $G_{8}$ is only and only Cayley graph of $A_{4}$.

Case6: Let $|G|=20$. By Lemmas 2.9 and 2.11, $G$ is a non-abelian group and so it is $D_{20}=D_{10} \times C_{2}, T_{20}$ or $F_{5,4}=\left\langle a, b \mid a^{5}=b^{4}=1, b^{-1} a b=a^{2}\right\rangle$. Since $\Gamma(S: G)$ is integral, $\Gamma(S: G)=G_{9}$ or $G_{10}$.

Let $G=F_{5,4}$. Since the graphs $G_{9}$ and $G_{10}$, does not have $C_{4}$ and $C_{5}$ as a subgraph, by Lemma 2.6, all of the elements of $S$ are of order 2 or 10. It is clear that $F_{5,4}$ does not have any element of order 10, so $S \subseteq \bar{b}$ (the bar indicates conjugacy class). By Lemma 2.3 and using characters table of $F_{5,4}$, we see that the eigenvalues of $\Gamma\left(S: F_{5,4}\right)$ corresponding to irreducible characters $\chi_{1}$ and $\chi_{3}$ are 3 . which is impossible.

Let $G=D_{10} \times C_{2}$ and $S_{1}=\left\{s_{1} \mid\left(s_{1}, c\right) \in S, c \in C_{2}\right\} \backslash\{1\}$, then by Lemma 2.10 and Case $4,\left|S_{1}\right|=2$ and so $-1 \leqslant \lambda_{i 0}$, where $\lambda_{i 0}$ is the eigenvalue of $\Gamma\left(S: D_{10} \times C_{2}\right)$ corresponding to a linear irreducible character of $D_{10} \times C_{2}$. This contradicts the fact that -3 is an eigenvalue of $G_{9}$ and $G_{10}$.

Let $G=T_{20}$. Since $a^{5} \in T_{20}$ is the unique element of order two and $G_{9}, G_{10}$, does not have $C_{4}$ and $C_{5}$ as a subgraph, $S=\left\{a^{5}, a^{r}, a^{-r}\right\}$. This contradicts the fact that $S$ generates $G$. Hence the graphs $G_{9}$ and $G_{10}$ are not Cayley graphs.

Case7: Let $|G|=24$. By Lemmas 2.9 and 2.11, $G$ is a non-abelian group and so $G=D_{12} \times C_{2}, T_{12} \times C_{2}, Q_{8} \times C_{3}, S L(2,3), D_{24}, T_{24}, U_{24}, V_{24}, S_{4}, D_{8} \times C_{3}, D_{6} \times C_{4}$ or $A_{4} \times C_{2}$. We show that $G_{13}$ is only and only Cayley graph of groups $S_{4}, A_{4} \times C_{2}, D_{8} \times C_{3}$, $D_{6} \times C_{4}$.

Let $G=S_{4}$. By Lemma 2.13, $\Gamma\left(S: S_{4}\right)=G_{13}$.

Let $G=A_{4} \times C_{2}$ and $S=\left\{\left(\left(\begin{array}{ll}1 & 2\end{array}\right)\left(\begin{array}{ll}3 & 4\end{array}\right), c\right),\left(\left(\begin{array}{lll}1 & 2 & 3\end{array}\right), c\right),\left(\left(\begin{array}{lll}1 & 3 & 2\end{array}\right), c\right)\right\}$, where $C_{2}=$ $\langle c\rangle$. Then by using the program written in Lemma 2.13, $\Gamma\left(S: A_{4} \times C_{2}\right)$ is integral with spectrum of $\left[-3,-2^{6},-1^{3}, 0^{4}, 1^{3}, 2^{6}, 3\right]$. So $\Gamma\left(S: A_{4} \times C_{2}\right)=G_{13}$.

Let $G=D_{8} \times C_{3}$ and $S=\left\{(a, c),\left(a^{3}, c\right),(b, 1)\right\}$, where $D_{8}=\langle a, b\rangle$ and $C_{2}=\langle c\rangle$. Then by using the program written in Lemma 2.13, $\Gamma\left(S: D_{8} \times C_{3}\right)$ is integral with spectrum of $\left[-3,-2^{6},-1^{3}, 0^{4}, 1^{3}, 2^{6}, 3\right]$ and so $\Gamma\left(S: D_{8} \times C_{3}\right)=G_{13}$.

Let $G=D_{6} \times C_{4}$. In the same manner we can see that $\Gamma\left(S: D_{6} \times C_{4}\right)=G_{13}$, where $D_{6}=\langle a, b\rangle, C_{4}=\langle c\rangle$ and $S=\left\{(a, c),\left(a^{3}, c\right),(b, 1)\right\}$.

It remains to prove that $\Gamma(S: G)$ is not integral, for others. On the contrary, let $\Gamma(S: G)=G_{13}$, for $G=Q_{8} \times C_{3}, T_{12} \times C_{2}, D_{12} \times C_{2}, T_{24}, D_{24}, S L(2,3)$ or $V_{24}$.

Let $G=Q_{8} \times C_{3}$ or $T_{12} \times C_{2}$ and $S_{1}=\left\{s_{1} \mid\left(s_{1}, c\right) \in S, c \in C_{3}\right\} \backslash\{1\}$ or $\left\{s_{1} \mid\left(s_{1}, c\right) \in\right.$ $\left.S, c \in C_{2}\right\} \backslash\{1\}$, then by Lemma 2.10 and Cases 3, 5, we have $\left|S_{1}\right|=2$ and so $-1 \leqslant \lambda_{i 0}$, where $\lambda_{i 0}$ is the eigenvalue of $\Gamma(S: G)$ corresponding to a linear irreducible character of $G$. This contradicts the fact that -3 is an eigenvalue of $G_{13}$.

Let $G=D_{12} \times C_{2}$ and $S_{1}=\left\{s_{1} \mid\left(s_{1}, c\right) \in S, c \in C_{2}\right\} \backslash\{1\}$. One can check that $(1, c) \in S$ where $C_{2}=\langle c\rangle$. So $\left|S_{1}\right|=2$ and $-1 \leqslant \lambda_{i 0}$, where $\lambda_{i 0}$ is the eigenvalue of $\Gamma\left(S: D_{12} \times C_{2}\right)$ corresponding to a linear irreducible character of $D_{12} \times C_{2}$. This contradicts the fact that -3 is an eigenvalue of $G_{13}$. 
Let $G=T_{24}$. Since $a^{6} \in T_{24}$ is the unique element of order two and $G_{13}$ does not have $C_{4}$ as a subgraph, $S=\left\{a^{6}, a^{r}, a^{-r}\right\}$ for $1 \leqslant r \leqslant 5$. This contradicts the fact that $S$ generates $G$.

Let $G=U_{24}=\left\langle a, b \mid a^{8}=b^{3}=1, a^{-1} b a=b^{-1}\right\rangle$. Since $a^{4}$ is the unique element of order two, $a^{4} \in S$ and so $a^{r} \notin S$ for $r \neq 4$ because of $S$ generates $G$. It is easy to see that $\left(a^{2 r} b\right)^{-1}=a^{8-2 r} b^{2}$ and $\left(a^{2 r+1} b\right)^{-1}=a^{8-2 r-1} b$. So $S=\left\{a^{4}, a^{2 r} b, a^{8-2 r} b^{2}\right\}$, $\left\{a^{4}, a^{2 r+1} b, a^{8-2 r-1} b\right\}$ or $\left\{a^{4}, a^{2 r+1} b^{2}, a^{8-2 r-1} b^{2}\right\}(0 \leqslant r \leqslant 3)$. If $S=\left\{a^{4}, a^{2 r} b, a^{8-2 r} b^{2}\right\}$, then by Lemma 2.3 and using characters table of $U_{6 n}$, the eigenvalue of $\Gamma\left(S: U_{24}\right)$ corresponding to $\chi_{4}$ is equal to 3 , which is not true. If $S=\left\{a^{4}, a^{2 r+1} b, a^{8-2 r-1} b\right\}$ or $\left\{a^{4}, a^{2 r+1} b^{2}, a^{8-2 r-1} b^{2}\right\}$, then by Lemma 2.3 and using characters table of $U_{6 n}$, the eigenvalue of $\Gamma\left(S: U_{24}\right)$ corresponding to $\chi_{1}$ is $-1+2 \cos ((2 r+1) \pi / 4)$ for $0 \leqslant r \leqslant 3$, obviously is not integer. Which is a contradiction.

Let $G=D_{24}$. First consider $a^{6} \in S$. Since $S$ generates $G, a^{r} \notin S$. By Lemma 2.3 , it is immediate that $\Gamma\left(S: D_{24}\right)=G_{13}$ does not have -3 as an eigenvalue, which is impossible. Thus $a^{6} \notin S$. Now suppose $a^{r} \in S$ where $1 \leqslant r \leqslant 5$. Since $S$ generates $D_{24},(r, 12)=1$. So $S=\left\{a^{r}, a^{-r}, a^{2 l} b\right\}$ or $\left\{a^{r}, a^{-r}, a^{2 l+1} b\right\}$ where $r=1$ or 5 . By Lemma 2.3 and using characters table of $D_{2 n}$, the sum of the eigenvalues of $\Gamma\left(S: D_{24}\right)$ corresponding to $\chi_{1}$ is $\sqrt{3}$ or $-\sqrt{3}$, which is impossible. Therefore, all of the elements of $S$ are in conjugacy class of $b$ or $a b$. Let $S=\left\{a^{2 s} b, a^{2 r+1} b, a^{2 l+1} b\right\}(1 \leqslant l, r, s \leqslant 5)$ and $\rho$ be an irreducible character of degree two of $D_{24}$. If $\lambda$ and $\mu$ are the eigenvalues $\Gamma\left(S: D_{24}\right)$ corresponding to $\rho$, then by Lemma 2.3 and using characters table of $D_{2 n}$, we have:

$\lambda+\mu=0$

$\lambda^{2}+\mu^{2}=6+2\left[\rho\left(a^{2 s-2 r-1}\right)+\rho\left(a^{2 s-2 l-1}\right)+\rho\left(a^{2 r-2 l}\right)\right]$.

A trivial verification shows that if $\omega=e^{\frac{2 \pi i}{12}}$, then $\omega+\omega^{-1}=\sqrt{3}, \omega^{2}+\omega^{-2}=1, \omega^{3}+\omega^{-3}=0$, $\omega^{4}+\omega^{-4}=-1$ and $\omega^{5}+\omega^{-5}=-\sqrt{3}$. From this and using characters table of $D_{2 n}$ we conclude that $\lambda^{2}+\mu^{2} \neq 0$. It follows that $\Gamma\left(S: D_{24}\right)$ does not have 0 as an eigenvalue. Therefore, $G_{13}$ does not have 0 as an eigenvalue, which is impossible.

Let $G=S L(2,3)$. It is easy to see that $g_{2}$ is the unique element of order two, so $g_{2} \in S$. On the other hand, since $g_{6} g_{7}=1$ and the graph $G_{13}$ does not have $C_{3}$ and $C_{4}$ as a subgraph, $S=\left\{g_{2}, x, x^{-1}\right\}$, such that $x$ is in conjugacy class of $g_{6}$ and $x^{-1}$ in conjugacy class of $g_{7}$. By Lemma 2.3 and using characters table of $S L(2,3)$, it is easily seen that the eigenvalues of corresponding to irreducible linear characters of $S L(2,3)$ are equal to zero. This contradicts the fact that -3 is an eigenvalue of $G_{13}$

Let $G=V_{24}=\left\langle a, b \mid a^{6}=b^{4}=(b a)^{2}=\left(a^{-1} b\right)^{2}=1\right\rangle$. Since the graph $G_{13}$ does not have $C_{3}$ and $C_{4}$ as a subgraph, $S \cap \bar{b}=\phi$ and $S \cap \overline{a^{2}}=\phi$ (the bar indicates conjugacy class). If $S \cap \overline{a b}=\phi$, then by Lemma 2.3 and using characters table of $V_{24}$, we see that the eigenvalues of corresponding to linear irreducible characters of $\chi_{1}$ and $\chi_{2}$ are equal to 3. Which is impossible. So $S \cap \overline{a b} \neq \phi$. Also if $b^{2} \in S$ or $a^{2} b^{2} \in S$, then by Lemma 2.3, we check at once that $\Gamma\left(S: V_{24}\right)$ does not have -3 as an eigenvalue, which is not true. Hence $S=\left\{a, a^{-1}, a^{r} b^{s}\right\},\left\{a b^{2}, a^{-1} b^{2}, a^{r} b^{s}\right\}$ or $\left\{a^{3}, a^{3} b^{2}, a^{r} b^{s}\right\}$, where $r \in\{1,3,5\}$ and $s \in\{1,3\}$. Let $\lambda$ and $\mu$ be the eigenvalues of $\Gamma\left(S: V_{24}\right)$ corresponding to irreducible character $\chi_{5}$. If $S=\left\{a^{3}, a^{3} b^{2}, a^{r} b^{s}\right\}$, then by Lemma 2.3 and using characters table of $V_{24}, \lambda+\mu=0$ and $\lambda^{2}+\mu^{2}=\chi_{5}\left(a^{6}\right)+\chi_{5}\left(a^{3} b^{2}\right)^{2}+\chi_{5}\left(a^{r} b^{s}\right)^{2}+2\left[\chi_{5}\left(a^{3} a^{3} b^{2}\right)+\chi_{5}\left(a^{r+3} b^{s}\right)+\chi_{5}\left(a^{r+3} b^{s+2}\right)\right]=10$. 
If $S=\left\{a, a^{-1}, a^{r} b^{s}\right\}$ or $\left\{a b^{2}, a^{-1} b^{2}, a^{r} b^{s}\right\}$, then by Lemma $2.3, \lambda+\mu=0$ and $\lambda^{2}+\mu^{2}=$ $\chi_{5}\left(a^{2}\right)+\chi_{5}\left(a^{-2}\right)+\chi_{5}\left(a^{r} b^{s}\right)^{2}+2\left[\chi_{5}\left(a a^{-1}\right)+\chi_{5}\left(a^{r+1} b^{s}\right)+\chi_{5}\left(a^{r-1} b^{s}\right)\right]$ or $\lambda+\mu=0$ and $\lambda^{2}+\mu^{2}=\chi_{5}\left(a b^{2}\right)^{2}+\chi_{5}\left(a^{-1} b^{2}\right)^{2}+\chi_{5}\left(a^{r} b^{s}\right)^{2}+2\left[\chi_{5}(1)+\chi_{5}\left(a^{r+1} b^{s+2}\right)+\chi_{5}\left(a^{r-1} b^{s+2}\right)\right]$, respectively.

By using character table of $V_{24}$, we have $\chi_{5}\left(a^{r+1} b^{s}\right)=\chi_{5}\left(a^{r-1} b^{s}\right)=\chi_{5}\left(a^{r+1} b^{s+2}\right)=$ $\chi_{5}\left(a^{r-1} b^{s+2}\right)=\chi_{5}\left(a^{r+3} b^{s}\right)=\chi_{5}\left(a^{r+3} b^{s+2}\right)=0$. So $\lambda^{2}+\mu^{2}=10$.

This gives $\lambda$ and $\mu$ are not integers, which is false.

Case8: Let $|G|=30$ and $\Gamma(S: G)=G_{6}$. By Lemmas 2.9 and 2.11, $G$ is a non-abelian group and so $G=D_{10} \times C_{3}, D_{30}$ or $U_{30}=\left\langle a, b \mid a^{10}=b^{3}=1, a^{-1} b a=b^{-1}\right\rangle$.

Let $G=D_{10} \times C_{3}$ and $S_{1}=\left\{s_{1} \mid\left(s_{1}, c\right) \in S, c \in C_{3}\right\} \backslash\{1\}$. By Lemma 2.10 and Case 4, $\left|S_{1}\right|=2$ and so $-1 \leqslant \lambda_{i 0}$, where $\lambda_{i 0}$ is the eigenvalue of $\Gamma\left(S: D_{10} \times C_{3}\right)$ corresponding to a linear irreducible character of $D_{10} \times C_{3}$. This contradicts the fact that -3 is an eigenvalue of $G_{6}$.

Let $\Gamma\left(S: D_{30}\right)=G_{6}$. By Lemma $2.12(\mathrm{ii})$, we have $4 \mid 10$, which is impossible.

Let $\Gamma\left(S: U_{30}\right)=G_{6}$. It is obvious that $U_{30}$ has exactly three elements of order two and they are $a^{5}, a^{5} b$ and $a^{5} b^{2}$. So $S \cap\left\{a^{5}, a^{5} b, a^{5} b^{2}\right\} \neq \phi$. If $S=\left\{a^{5}, a^{5} b, a^{5} b^{2}\right\}$, then by Lemma 2.3 and using characters table of $U_{6 n}$, the eigenvalues of $\Gamma\left(S: U_{30}\right)$ corresponding to irreducible characters $\chi_{1}$ and $\chi_{5}$ are -3 . This contradicts the fact that the multiplicity -3 as an eigenvalue of $G_{6}$ is one. If $a^{2 r} \in S$ or $a^{2 r} b \in S(0 \leqslant r \leqslant 4)$, then by Lemma 2.3 and using characters table of $U_{6 n}$, the eigenvalue of $\Gamma\left(S: U_{30}\right)$ corresponding to irreducible character $\chi_{5}$ is 1 , this show that 1 is an eigenvalue of $G_{6}$, which is not true. Thus $S=\left\{a^{5} b^{k}, a^{2 r+1} b^{s},\left(a^{2 r+1} b^{s}\right)^{-1}\right\}$, where $k, s \in\{0,1,2\}$ and $r \in\{0,1,3,4\}$. By Lemma 2.3 and using characters table of $U_{6 n}$, the eigenvalue of $\Gamma\left(S: U_{30}\right)$ corresponding to irreducible character $\chi_{1}$ is $-1+2 \cos ((2 r+1) \pi / 5)$ for $r \in\{0,1,3,4\}$. This is not integer. which is a contradiction. Therefor $G_{6}$ is not Cayley graph.

Hence there are exactly seven connected, cubic integral Cayley graphs. This proves the theorem.

Theorem 3.1 (See [14]) Let $C_{n}=\langle a\rangle$. If $S=\left\{a^{j} \mid j \in B(1, n)\right\}$, then $\Gamma\left(S: C_{n}\right)$ is integral.

Proof. By Lemma 2.2, $\Gamma\left(S: C_{n}\right)$ is connected graph. By Lemmas 2.3 and 2.4, $n$ eigenvalues of $\Gamma\left(S: C_{n}\right)$ are $\lambda_{r}=\sum_{j \in B(1, n)} \omega^{j r},(1 \leqslant r<n)$. By equation $(2.1), \lambda_{r}=C(r, n)$, $(1 \leqslant r<n)$. Hence $\Gamma\left(S: C_{n}\right)$ is integral.

Corollary 3.2 For any natural number $n$, there is at least an connected, $\varphi(n)$-regular integral graph with $n$ vertices.

Theorem 3.3 Let $C_{n}=\langle a\rangle, d \mid n(1<d<n)$ and $A_{d}=\left\{a^{d j} \mid j \in B\left(1, \frac{n}{d}\right)\right\}$. If $S=A_{1} \cup A_{d}$, then $\Gamma\left(S: C_{n}\right)$ is integral.

Proof. By Lemma 2.2, $\Gamma\left(S: C_{n}\right)$ is connected graph. Let $\lambda_{r}(0 \leqslant r \leqslant n-1)$ be the eigenvalues of $\Gamma\left(S: C_{n}\right)$. By Lemmas 2.3 and 2.4, we have: 
$\lambda_{r}=\sum_{g \in A_{1}} \rho_{r}(g)+\sum_{g \in A_{d}} \rho_{r}(g)=\sum_{j \in B(1, n)} \rho_{r}\left(a^{j}\right)+\sum_{j \in B\left(1, \frac{n}{d}\right)} \rho_{r}\left(a^{d j}\right)=\sum_{j \in B(1, n)} \omega^{j r}+\sum_{j \in B\left(1, \frac{n}{d}\right)} \omega^{d j r}$.

By equation $(2.1), \sum_{j \in B(1, n)} \omega^{j r}$ and $\sum_{j \in B\left(1, \frac{n}{d}\right)} \omega^{d j r}$ are integer. Hence $\Gamma\left(S: C_{n}\right)$ is integral.

Corollary 3.4 For any natural number $n$, there is at least a connected, $\left(\varphi(n)+\varphi\left(\frac{n}{d}\right)\right)$ regular integral graph with $n$ vertices, where $d \mid n(1<d<n)$.

Lemma 3.5 Let $G=C_{m} \times C_{n}, C_{m}=\langle a\rangle$ and $C_{n}=\langle b\rangle$ so that $(m, n) \neq 1$. If $S=$ $\left\{\left(a^{j}, b^{j^{\prime}}\right) \mid j \in B(1, m), j^{\prime} \in B(1, n)\right\} \cup\left\{\left(a^{j}, 1\right) \mid j \in B(1, m)\right\} \cup\left\{\left(1, b^{j^{\prime}}\right) \mid j^{\prime} \in B(1, n)\right\}$, then $\Gamma(S: G)$ is integral.

Proof. It is clear that $\Gamma(S: G)$ is connected graph. By Lemma 2.3, $m n$ eigenvalues of $\Gamma(S: G)$ are $\lambda_{k r}=\sum_{g \in S} \rho_{k r}(g),(0 \leqslant k \leqslant m-1)$ and $(0 \leqslant r \leqslant n-1)$. By Lemma 2.5, $\lambda_{k r}=\sum_{j \in B(1, m)}\left(\sum_{j^{\prime} \in B(1, n)} \omega_{1}^{k j} \omega_{2}^{r j^{\prime}}\right)+\sum_{j \in B(1, m)} \omega_{1}^{k j}+\sum_{j^{\prime} \in B(1, n)} \omega_{2}^{r j^{\prime}}$.

An easy computation shows:

$\lambda_{k r}=\sum_{j \in B(1, m)} \omega_{1}^{k j} \sum_{j^{\prime} \in B(1, n)} \omega_{2}^{r j^{\prime}}+\sum_{j \in B(1, m)} \omega_{1}^{k j}+\sum_{j^{\prime} \in B(1, n)} \omega_{2}^{r j^{\prime}}$. By equation $(2.1), \sum_{j \in B(1, m)} \omega_{1}^{k j}$ and $\sum_{j^{\prime} \in B(1, n)} \omega_{2}^{r j^{\prime}}$ are integer. Hence $\Gamma(S: G)$ is integral.

Theorem 3.6 Let $G=C_{n_{1}} \times \ldots \times C_{n_{l}}$ and $C_{n_{i}}=\left\langle a_{i}\right\rangle$, so that for any $i, j \in\{1, \ldots, l\}$, $\left(n_{i}, n_{j}\right) \neq 1$. If $S=\left\{\left(a_{1}^{j_{1}}, a_{2}^{j_{2}}, \ldots, a_{l}^{j_{l}}\right) \mid j_{i} \in B\left(1, n_{i}\right), i=1, \ldots, l\right\} \cup\left\{\left(a_{1}^{j_{1}}, 1, \ldots, 1\right) \mid j_{1} \in\right.$ $\left.B\left(1, n_{1}\right)\right\} \cup \ldots \cup\left\{\left(1,1, \ldots, a_{l}^{j_{l}}\right) \mid j_{l} \in B\left(1, n_{l}\right)\right\}$, then $\Gamma(S: G)$ is integral.

Proof. Suppose $\alpha=\sum_{j_{1} \in B\left(1, n_{1}\right)} \cdots \sum_{j_{l} \in B\left(1, n_{l}\right)} \omega_{1}^{r_{1} j_{1}} \cdots \omega_{l}^{r_{l} j_{l}}$, where $\omega_{t}=e^{\frac{2 \pi i}{n_{t}}}$, for $t=$ $1, \ldots, l$. One can check that $\alpha=\left(\sum_{j_{1} \in B\left(1, n_{1}\right)} \omega_{1}^{r_{1} j_{1}}\right) \cdots\left(\sum_{j_{l} \in B\left(1, n_{l}\right)} \omega_{l}^{r_{l} j_{l}}\right)$. By Lemma 2.3, $n_{1} n_{2} \ldots n_{l}$ eigenvalues of $\Gamma(S: G)$ are $\lambda_{r_{1} \ldots r_{l}}=\sum_{g \in S} \rho_{r_{1} r_{2} \ldots r_{l}}(g)$, where $0 \leqslant r_{i} \leqslant n_{i}-1$ and $1 \leqslant i \leqslant l$. By Lemma 2.5, $\lambda_{r_{1} \ldots r_{l}}=\alpha+\sum_{j_{1} \in B\left(1, n_{1}\right)} \omega_{1}^{r_{1} j_{1}}+\ldots+\sum_{j_{l} \in B\left(1, n_{l}\right)} \omega_{l}^{r_{l} j_{l}}$.

By equation $(2.1), \sum_{j_{i} \in B\left(1, n_{i}\right)} \omega_{i}^{r_{i} j_{i}},(1 \leqslant i \leqslant l)$ is integer. Hence $\Gamma(S: G)$ is integral.

Corollary 3.7 Let $n=n_{1} \cdots n_{l}$ such that $\left(n_{i}, n_{j}\right) \neq 1$, where $1 \leqslant i, j \leqslant l$. Then there is at least a connected $\left(\sum_{i=1}^{l} \varphi\left(n_{i}\right)\right)\left(\prod_{i=1}^{l} \varphi\left(n_{i}\right)\right)$-regular integral graph with $n$ vertices.

Theorem 3.8 Let $D_{2 n}=\left\langle a, b \mid a^{n}=b^{2}=1,(a b)^{2}=1\right\rangle, n=2 m+1$ ( $\left.n \neq 1\right)$ and $S=\left\{a^{k} \mid k \in B(1, n)\right\} \cup\left\{b a^{k} \mid k \in B(1, n)\right\}$. Then $\Gamma\left(S: D_{2 n}\right)$ is integral. 
Proof.Since $S$ generates $D_{2 n}, \Gamma\left(S: D_{2 n}\right)$ is connected graph. We know that $\{1\}$, $\left\{a^{r}, a^{-r}\right\}, 1 \leqslant r \leqslant(n-1) / 2$ and $\left\{a^{s} b \mid 0 \leqslant s \leqslant n-1\right\}$ are the conjugacy classes of $D_{2 n}$. Let $A_{j}=\sum_{k \in B(1, n)} \omega^{j k}, S_{1}=\left\{a^{k} \mid k \in B(1, n)\right\}$ and $S_{2}=\left\{b a^{k} \mid k \in B(1, n)\right\}$. If $\lambda_{j 1}$, $\lambda_{j 2}$ (Each one 2 times)for $1 \leqslant j \leqslant m, \lambda_{m+1}$ and $\lambda_{m+2}$ are $2 n$ eigenvalues of $\Gamma\left(S: D_{2 n}\right)$, then by Proposition 4.1 from [2], $\lambda_{j 1}+\lambda_{j 2}=2 A_{j}$ and $\lambda_{j 1}^{2}+\lambda_{j 2}^{2}=4 A_{j}^{2}$. So $\lambda_{j 1}=0$, $\lambda_{j 2}=2 A_{j}$ or $\lambda_{j 1}=2 A_{j}, \lambda_{j 2}=0$. Also $\lambda_{m+1}=0$ and $\lambda_{m+2}=\left|S_{1}\right|+\left|S_{2}\right|=2 \varphi(n)$. By equation (2.1), $A_{j}$ is integer. So all of the eigenvalues of $\Gamma\left(S: D_{2 n}\right)$ are integers. Hence $\Gamma\left(S: D_{2 n}\right)$ is integral.

Corollary 3.9 For any odd natural number $n(n \neq 1)$, there is at least a connected, $(2 \varphi(n))$-regular integral graph with $2 n$ vertices.

Proof of Theorem 1.2. It is clear that $\Gamma\left(S: D_{2 n}\right)$ is connected graph. Let $C_{1}=$ $\{k \mid k \in B(1, n)\}=\left\{k_{1}, \ldots, k_{\varphi(n)}\right\}$ and $C_{2}=\left\{d k \mid k \in B\left(1, \frac{n}{d}\right)\right\}=\left\{k_{1}^{\prime}, \ldots, k_{\varphi\left(\frac{n}{d}\right)}^{\prime}\right\}$. Then $C_{1} \cap C_{2}=\phi$. Suppose $C_{1} \cup C_{2}=\left\{k_{i} \mid 1 \leqslant k_{1}<\cdots<k_{t} \leqslant n-1\right\}$ and $A_{j}=\sum_{u=1}^{t} \omega^{j k_{u}}$ for $j=1, \ldots, m$. Then $A_{j}=\sum_{k \in C_{1}} \omega^{j k}+\sum_{k^{\prime} \in C_{2}} \omega^{j k^{\prime}}$ and by equation (2.1), $A_{j}$ is integer. If $\lambda_{j 1}, \lambda_{j 2}$ for $1 \leqslant j \leqslant m$ (Each one 2 times), $\lambda_{m+1}$ and $\lambda_{m+2}$ are $2 n$ eigenvalues of $\Gamma\left(S: D_{2 n}\right)$, then by Proposition 4.1 from [2], we have $\lambda_{j 1}+\lambda_{j 2}=2 A_{j}$ and $\lambda_{j 1}^{2}+\lambda_{j 2}^{2}=4 A_{j}^{2}$. So $\lambda_{j 1}=0, \lambda_{j 2}=2 A_{j}$ or $\lambda_{j 1}=2 A_{j}, \lambda_{j 2}=0$. Also $\lambda_{m+1}=0$ and $\lambda_{m+2}=2\left|C_{1}\right|+2\left|C_{2}\right|=2 \varphi(n)+2 \varphi\left(\frac{n}{d}\right)$. Since $A_{j}$ is integer, $2 n$ eigenvalues of $\Gamma\left(S: D_{2 n}\right)$ are integers. Hence $\Gamma\left(S: D_{2 n}\right)$ is integral.

Corollary 3.10 For any odd natural number $n$, there is at least a connected, $(2 \varphi(n)+$ $\left.2 \varphi\left(\frac{n}{d}\right)\right)$-regular integral graph with $2 n$ vertices, where $d \mid n(1<d<n)$.

Proof of Theorem 1.3. We know that $\{1\},\left\{a^{r}, a^{-r}\right\},(1 \leqslant r \leqslant n-1),\left\{a^{2 k} b \mid 0 \leqslant k \leqslant\right.$ $n-1\}$ and $\left\{a^{2 k+1} b \mid 0 \leqslant k \leqslant n-1\right\}$ are all of the conjugacy classes of $T_{4 n}$. It is clear that $T_{4 n}=\langle S\rangle, S=S^{-1}$ and $1 \notin S$. Let $\lambda_{j 1}, \lambda_{j 2}$ for $1 \leqslant j \leqslant n-1$ (Each one 2 times) and $\mu_{l}$ for $1 \leqslant l \leqslant 4$ be $4 n$ eigenvalues of $\Gamma\left(S: T_{4 n}\right)$. Then by Lemma 2.3 and using characters table of $T_{4 n}$, we have:

$$
\begin{aligned}
& \mu_{1}=\sum_{g \in S} \chi_{1}(g)=2 n, \mu_{2}=\sum_{g \in S} \chi_{2}(g)=0, \mu_{3}=\sum_{g \in S} \chi_{3}(g)=2 n-4 \text { and } \mu_{4}=\sum_{g \in S} \chi_{4}(g)=0 . \\
& \lambda_{j 1}+\lambda_{j 2}=\sum_{g \in S} \rho_{j}(g)=2 \sum_{k=1}^{n-1} \rho_{j}\left(a^{k}\right)=2 \sum_{k=1}^{n-1} \omega^{j k}+\omega^{-j k} \\
& \lambda_{j 1}^{2}+\lambda_{j 2}^{2}=\sum_{s_{1}, s_{2} \in S} \rho_{j}\left(s_{1} s_{2}\right)=(4 n-8) \sum_{k=1}^{n-1} \rho_{j}\left(a^{k}\right)+2 n\left[\rho_{j}\left(a^{n}\right)+\rho_{j}(1)\right]= \\
& (4 n-8) \sum_{k=1}^{n-1}\left(\omega^{j k}+\omega^{-j k}\right)+2 n\left[2(-1)^{j}+2\right] .
\end{aligned}
$$

By Lemma 2.1, if $j$ is odd, then $\lambda_{j 1}+\lambda_{j 2}=0$ and $\lambda_{j 1}^{2}+\lambda_{j 2}^{2}=0$ and so $\lambda_{j 1}=\lambda_{j 2}=0$ (Each one two times). 
If $j$ is even, then $\lambda_{j 1}+\lambda_{j 2}=-4$ and $\lambda_{j 1}^{2}+\lambda_{j 2}^{2}=16$ and so $\lambda_{j 1}=0$ and $\lambda_{j 2}=-4$ (Each one two times). Therefore, the spectrum of $\Gamma\left(S: T_{4 n}\right)$ is: $\left[-4^{n-1}, 0^{3 n-1}, 2 n-4,2 n\right]$.

Corollary 3.11 For any odd natural number $n,(n \neq 1)$, there is at least a connected, $2 n$-regular integral graph with 4 n vertices.

Proof of Theorem 1.4. Consider $A=\left\{a^{2 k} b \mid 1 \leqslant k \leqslant n-1\right\} \cup\left\{a^{2 k} b^{2} \mid 1 \leqslant\right.$ $k \leqslant n-1\}$ and $B=\left\{a^{2 k+1} b \mid 0 \leqslant k \leqslant n-1\right\}$. We know that $\left\{a^{2 r}\right\},\left\{a^{2 r} b, a^{2 r} b^{2}\right\}$ and $\left\{a^{2 r+1}, a^{2 r+1} b, a^{2 r+1} b^{2}\right\},(0 \leqslant r \leqslant n-1)$ are the conjugacy classes of $U_{6 n}$. An easy computation shows that $b a^{2}=a^{2} b,\left(a^{2 r} b\right)^{-1}=a^{-2 r} b^{2},\left(a^{2 r+1} b^{2}\right)^{-1}=a^{-2 r-1} b^{2}$ and $\left(a^{2 r+1} b\right)^{-1}=a^{-2 r-1} b$. So $U_{6 n}=\langle S\rangle, S=S^{-1}$ and $1 \notin S$. Let $\lambda_{j 1}, \lambda_{j 2}$ for $0 \leqslant j \leqslant n-1$ (Each one 2 times) and $\mu_{l}$ for $0 \leqslant l \leqslant 2 n-1$ be $6 n$ eigenvalues of $\Gamma\left(S: U_{6 n}\right)$ corresponding to the characters of $\rho_{j}$ and $\chi_{l}$ of $U_{6 n}$, respectively. Then by Lemma 2.3 and using characters table of $U_{6 n}$, we have:

$\mu_{0}=\sum_{s \in S} \chi_{0}(s)=3 n-2, \mu_{n}=\sum_{s \in S} \chi_{n}(s)=n-2$,

$\mu_{l}=\sum_{s \in S} \chi_{l}(s)=-2$, for $1 \leqslant l \leqslant 2 n-1$ and $l \neq n$.

Also for $0 \leqslant j \leqslant n-1$, we have:

$\lambda_{j 1}^{2}+\lambda_{j 2}^{2}=\sum_{s_{1}, s_{2} \in A} \rho_{j}\left(s_{1} s_{2}\right)+\sum_{s_{1}, s_{2} \in B} \rho_{j}\left(s_{1} s_{2}\right)+\sum_{s_{1} \in A, s_{2} \in B}\left(\rho_{j}\left(s_{1} s_{2}\right)+\rho_{j}\left(s_{2} s_{1}\right)\right)$.

One can check that :

$\sum_{s_{1}, s_{2} \in A} \rho_{j}\left(s_{1} s_{2}\right)=(2 n-4)\left[\sum_{k=1}^{n-1}-\omega^{2 k j}+\sum_{k=1}^{n-1} 2 \omega^{2 k j}\right]+(2 n-2)\left[2+\sum_{k=1}^{n-1}-\omega^{2 k j}\right]$.

$\sum_{s_{1}, s_{2} \in B} \rho_{j}\left(s_{1} s_{2}\right)=n \sum_{k=1}^{n-1} 2 \omega^{2 k j}$.
$\sum_{s_{1} \in A, s_{2} \in B}\left(\rho_{j}\left(s_{1} s_{2}\right)+\rho_{j}\left(s_{2} s_{1}\right)\right)=0$.

By Lemma 2.1, $\lambda_{01}^{2}+\lambda_{02}^{2}=4 n^{2}-4 n+2$ and $\lambda_{j 1}^{2}+\lambda_{j 2}^{2}=2$ for $1 \leqslant j \leqslant n-1$. On the other hand, it is clear that $\lambda_{01}+\lambda_{02}=\sum_{s \in S} \rho_{0}(s)=-2 n+2$ and $\lambda_{j 1}+\lambda_{j 2}=\sum_{s \in S} \rho_{j}(s)=2$ for $1 \leqslant j \leqslant n-1$. So $\lambda_{01}=1$ and $\lambda_{02}=1-2 n$ (Each one two times).

Also $\lambda_{j 1}=1=\lambda_{j 2}$ for $1 \leqslant j \leqslant n-1$ (Each one two times). Therefore, the spectrum of $\left.\Gamma\left(S: U_{6 n}\right)\right)$ is: $\left[(1-2 n)^{2},-2^{2 n-2}, 1^{4 n-2}, n-2,3 n-2\right]$.

Corollary 3.12 For any odd natural number $n(n \neq 1)$, there is at least a connected $(3 n-2)$-regular integral graph with $6 n$ vertices.

\begin{tabular}{cccccc}
\multicolumn{6}{c}{ Character Table of $Q_{8}$} \\
\hline & 1 & $a^{2}$ & $a$ & $b$ & $a b$ \\
\hline$\chi_{1}$ & 1 & 1 & 1 & 1 & 1 \\
$\chi_{2}$ & 1 & 1 & 1 & -1 & -1 \\
$\chi_{3}$ & 1 & 1 & -1 & 1 & -1 \\
$\chi_{4}$ & 1 & 1 & -1 & -1 & 1 \\
$\chi_{5}$ & 2 & -2 & 0 & 0 & 0 \\
\hline
\end{tabular}

THE ELECTRONiC JouRnal of COMBinatorics 16 (2009), \#R122 
Character Table of $A_{4}$

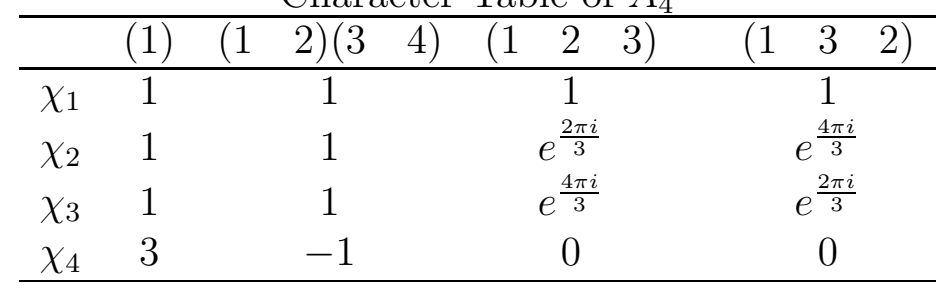

Character Table of $F_{5,4}$

\begin{tabular}{cccccc}
\hline & 1 & $a$ & $b$ & $b^{2}$ & $b^{3}$ \\
\hline$\chi_{1}$ & 1 & 1 & 1 & 1 & 1 \\
$\chi_{2}$ & 1 & 1 & $i$ & -1 & $-i$ \\
$\chi_{3}$ & 1 & 1 & -1 & 1 & -1 \\
$\chi_{4}$ & 1 & 1 & $-i$ & -1 & $i$ \\
$\chi_{5}$ & 4 & -1 & 0 & 0 & 0 \\
\hline
\end{tabular}

Character Table of $S L(2,3)$

\begin{tabular}{cccccccc}
\hline & $g_{1}=1$ & $g_{2}$ & $g_{3}$ & $g_{4}$ & $g_{5}$ & $g_{6}$ & $g_{7}$ \\
\hline$\chi_{1}$ & 1 & 1 & 1 & 1 & 1 & 1 & 1 \\
$\chi_{2}$ & 1 & 1 & 1 & $e^{\frac{2 \pi i}{3}}$ & $e^{\frac{4 \pi i}{3}}$ & $e^{\frac{4 \pi i}{3}}$ & $e^{\frac{2 \pi i}{3}}$ \\
$\chi_{3}$ & 1 & 1 & 1 & $e^{\frac{4 \pi i}{3}}$ & $e^{\frac{2 \pi i}{3}}$ & $e^{\frac{2 \pi i}{3}}$ & $e^{\frac{4 \pi i}{3}}$ \\
$\chi_{4}$ & 3 & 3 & -1 & 0 & 0 & 0 & 0 \\
$\chi_{5}$ & 2 & -2 & 0 & -1 & -1 & 1 & 1 \\
$\chi_{6}$ & 2 & -2 & 0 & $-e^{\frac{2 \pi i}{3}}$ & $-e^{\frac{4 \pi i}{3}}$ & $e^{\frac{4 \pi i}{3}}$ & $e^{\frac{2 \pi i}{3}}$ \\
$\chi_{7}$ & 2 & -2 & 0 & $-e^{\frac{4 \pi i}{3}}$ & $-e^{\frac{2 \pi i}{3}}$ & $e^{\frac{2 \pi i}{3}}$ & $e^{\frac{4 \pi i}{3}}$ \\
\hline
\end{tabular}

$$
\begin{aligned}
g_{2}=\left(\begin{array}{cc}
-1 & 0 \\
0 & -1
\end{array}\right), g_{3}=\left(\begin{array}{cc}
0 & 1 \\
-1 & 0
\end{array}\right), \\
g_{5}^{-1}=g_{4}=\left(\begin{array}{ll}
1 & 1 \\
0 & 1
\end{array}\right), g_{7}^{-1}=g_{6}=\left(\begin{array}{cc}
-1 & 1 \\
0 & -1
\end{array}\right)
\end{aligned}
$$

Character Table of $V_{24}$

\begin{tabular}{cccccccccc}
\hline & 1 & $b^{2}$ & $a$ & $a^{3}$ & $a^{5}$ & $a^{2}$ & $a^{2} b^{2}$ & $b$ & $a b$ \\
\hline$\chi_{1}$ & 1 & 1 & 1 & 1 & 1 & 1 & 1 & 1 & 1 \\
$\chi_{2}$ & 1 & 1 & 1 & 1 & 1 & 1 & 1 & -1 & -1 \\
$\chi_{3}$ & 1 & 1 & -1 & -1 & -1 & 1 & 1 & 1 & -1 \\
$\chi_{4}$ & 1 & 1 & -1 & -1 & -1 & 1 & 1 & -1 & 1 \\
$\chi_{5}$ & 2 & -2 & 0 & 0 & 0 & 2 & -2 & 0 & 0 \\
$\chi_{6}$ & 2 & -2 & $i \sqrt{3}$ & 0 & $-i \sqrt{3}$ & -1 & 1 & 0 & 0 \\
$\chi_{7}$ & 2 & -2 & $-i \sqrt{3}$ & 0 & $i \sqrt{3}$ & -1 & 1 & 0 & 0 \\
$\chi_{8}$ & 2 & 2 & 1 & -2 & 1 & -1 & -1 & 0 & 0 \\
$\chi_{9}$ & 2 & 2 & -1 & 2 & -1 & -1 & -1 & 0 & 0 \\
\hline
\end{tabular}


Character Table of $D_{2 n}, n=2 m+1$ odd

\begin{tabular}{llll}
\hline & 1 & $a^{r}$ & $b$ \\
\hline$\chi_{j}$ & 2 & $\omega^{j r}+\omega^{-j r}$ & 0 \\
$\chi_{m+1}$ & 1 & 1 & -1 \\
$\chi_{m+2}$ & 1 & 1 & 1 \\
\hline
\end{tabular}

$\omega=e^{\frac{2 \pi i}{n}}, 1 \leqslant j \leqslant m$ and $1 \leqslant r \leqslant m$

Character Table of $D_{2 n}, n=2 m$ even

\begin{tabular}{llllll}
\hline & 1 & $a^{m}$ & $a^{r}$ & $b$ & $a b$ \\
\hline$\chi_{j}$ & 2 & $2(-1)^{j}$ & $\omega^{j r}+\omega^{-j r}$ & 0 & 0 \\
$\chi_{m+1}$ & 1 & 1 & 1 & -1 & -1 \\
$\chi_{m+2}$ & 1 & $(-1)^{m}$ & $(-1)^{r}$ & 1 & -1 \\
$\chi_{m+3}$ & 1 & $(-1)^{m}$ & $(-1)^{r}$ & -1 & 1 \\
$\chi_{m+4}$ & 1 & 1 & 1 & 1 & 1 \\
\hline$\omega=e^{\frac{2 \pi r}{n}}, 1 \leqslant j \leqslant m$ and $1 \leqslant r \leqslant m-1$ & & & & &
\end{tabular}

Character Table of $T_{4 n}, n=2 m+1$ odd

\begin{tabular}{llrrr}
\hline Characters & $a^{n}$ & $a^{r}$ & $a^{2 r} b$ & $a^{2 r+1} b$ \\
\hline$\chi_{1}$ & 1 & 1 & 1 & 1 \\
$\chi_{2}$ & -1 & $(-1)^{r}$ & $i$ & $-i$ \\
$\chi_{3}$ & 1 & 1 & -1 & -1 \\
$\chi_{4}$ & -1 & $(-1)^{r}$ & $-i$ & $i$ \\
$\rho_{j}$ & $2(-1)^{j}$ & $\omega^{j r}+\omega^{-j r}$ & 0 & 0 \\
\hline
\end{tabular}

$\omega=e^{\frac{2 \pi i}{2 n}}, 1 \leqslant j \leqslant n-1$ and $0 \leqslant r \leqslant n-1$

Character Table of $T_{4 n}, n=2 m$ even

\begin{tabular}{|c|c|c|c|c|}
\hline & $a^{n}$ & $a^{r}$ & $a^{2 r} b$ & $a^{2 r+1} b$ \\
\hline$\chi_{1}$ & 1 & 1 & 1 & 1 \\
\hline$\chi_{2}$ & 1 & 1 & -1 & -1 \\
\hline$\chi_{3}$ & 1 & $(-1)^{r}$ & 1 & -1 \\
\hline$\chi_{4}$ & 1 & $(-1)^{r}$ & -1 & 1 \\
\hline$\rho_{j}$ & $2(-1)^{j}$ & $\omega^{j r}+\omega^{-j r}$ & 0 & 0 \\
\hline
\end{tabular}

$\omega=e^{\frac{2 \pi i}{2 n}}, 1 \leqslant j \leqslant n-1$ and $0 \leqslant r \leqslant n-1$

Character Table of $U_{6 n}$

\begin{tabular}{llll}
\hline & $a^{2 r}$ & $a^{2 r} b$ & $a^{2 r+1}$ \\
\hline$\chi_{l}$ & $\omega^{2 l r}$ & $\omega^{2 l r}$ & $\omega^{2 l r+l}$ \\
$\rho_{j}$ & $2 \omega^{2 j r}$ & $-\omega^{2 j r}$ & 0 \\
\hline$\omega=e^{\frac{2 \pi i}{2 n}}, 0 \leqslant l \leqslant 2 n-1,0 \leqslant j \leqslant n-1$ and $0 \leqslant r \leqslant n-1$ & & &
\end{tabular}

Acknowledgments. The authors are very grateful to the referee for his/her useful comments. This research was partially supported by the Center of Excellence for Mathematics, University of Isfahan. 


\section{References}

[1] O. Ahmadi, N. Alon, I.F. Blake, I.E. Shparlinski, Graphs with integral spectrum, Linear Alg. Appl., 430 (2009), 547-552.

[2] L. Babai, Spectra of Cayley graphs, J. Combin. Theory Ser. B, 27 (1979), 180-189.

[3] K. Balińska, D. Cvetković, M. Lepović, S. Simić, There are exactly 150 connected integral graphs up to 10 vertices, Univ. Beograd. Publ. Elektrotehn. Fak., Ser. Mat., 10 (1999) 95-105.

[4] K.T. Balińska, M. Kupczyk, S.K. Simić, K.T. Zwierzyński, On generating all integral graphs on 11 vertices, The Technical University of Poznan, Computer Science Center Report No. 469, 1999/2000.

[5] K. Balińska, D. Cvetković, Z. Rodosavljevic, S. Simić, D. Stevanovic, A survey on integral graphs, Univ. Beograd, Publ. Elektrotehn. Fak. Ser. Mat. 13 (2003), 42-65.

[6] N. Biggs, Algebraic Graph Theory, Cambridge University Press, Cambridge, 1993.

[7] F. C. Bussemaker, D. Cvetković, There are exactly 13 connected, cubic, integral graphs., Univ. Beograd, Publ. Elektrotehn. Fak., Ser. Mat., Fiz., Nos. 544-576 (1976), 43-48.

[8] D.M. Cvetković, M. Doob, H. Sachs, Spectra of graphs - Theory and applications, 3rd edition, Johann Ambrosius Barth Verlag, Heidelberg-Leipzig, 1995.

[9] D. Cvetković, Cubic integral graphs, Univ. Beograd, Publ. Elektrotehn. Fak., Ser. Mat., Fiz., Nos. 498-541 (1975), 107-113.

[10] H.D. Friedman, On the impossibility of certain Moore graphs, J. Combin. Theory Ser. B, 10 (1971), 245-252.

[11] J. Friedman, On Cayley graphs on the symmetric group generated by transpositions, Combinatorica, 20 (2000), 505-519.

[12] F. Harary, A.J. Schwenk, Which graphs have integral spectra?, Graphs and Combinatorics, 390 (1974), 45-51.

[13] G. James, M. Liebeck, Representations and Characters of groups, Cambridge University Press, Cambridge, 1993.

[14] W. Klotz, T. Sander, Some properties of unitary Cayley graphs, Electronic Journal of Combinatorics, 14 (2007), no. 1. Research Paper 45, 12 pp.

[15] P.J. McCarthy, Introduction to arithmetical functions, Springer, New York, 1986.

[16] A.J. Schwenk, Exactly thirteen connected cubic graphs have integral spectra, Theory and applications of graphs (Proc. Internat. Conf., Western Mich. Univ., Kalamazoo, Mich., 1976), pp. 516-533, Lecture Notes in Math., 642, Springer, Berlin, 1978.

[17] W. So, Integral circulant graphs, Discrete Mathematics 306 (2006) 153-158.

[18] S. Ramanujan, On certain trigonometrical sums and their applications in the theory of numbers, [Trans. Cambridge Philos. Soc. 22 (1918), no. 13, 259-276]. Collected papers of Srinivasa Ramanujan, 179-199, AMS Chelsea Publ., Providence, RI, 2000.

[19] The GAP Group, GAP-Groups, Algoritms, and Programing, Version 4.4.4, 2004, http://gap-system.org. 